[11] Golinko I.M. Dinamichna model teplomasoobminnih protsesiv u kameri forsunochnogo zvolozhennya / I.M. Golinko, A.I. Kubrak, I.E. Galitska // Informatsiyni sistemi, mehanika ta keruvannya. 2014. № 11, S. 61-71.;

[12] Golinko I.M. Kompleksna dinamichna model promislovogo konditsionera iz forsunochnim zvolozhuvachem $\mathrm{u}$ prostori stanu / I.M. Golinko, I.E. Galitska // Girotehnologiyi, navigatsiya, keruvannya ruhom i konstruyuvannya aviatsiyno;

[13] kosmichnoyi tehniki: zbirka dopovidey H Mizhnarodnoyi naukovo-praktichnoyi konferentsiyi, 16 - 17 kvitnya 2015 r., - Kiyiv, 2015. S. 201-207.;

[14] Katalog. VENTUS - ventilyatsionnyie agregatyi i tsentralnyie konditsioneryi. -M.: VTSGroup, 2003. -156 c.;

[15] Kuo B. Teoriya i proektirovanie tsifrovyih sistem upravleniya: per. s angl. -M.:Mashinostroenie, 1986. -448 s.;

[16] Rey U. Metodyi upravleniya tehnologicheskimi protsessami / U. Rey. -M.: Mir, 1983. -368 s.;

[17] Golinko I.M. Analiz sistemi keruvannya dlya promislovogo konditsionera iz parozvolozhuvachem / I.M. Golinko, V.G. Tregub // Shidno-Evropeyskiy zhurnal peredovih tehnologiy. -2012, № 6/8(60). -S. 14-18.;

[18] Krasnov Yu.S. Sistemyi ventilyatsii i konditsionirovaniya / Yu.S. Krasnov, A.P. Borisoglebskaya, A.V. Anti-pov. M.: TermoKul, 2004. -373 s.

\title{
АППРОКСИМАЦИЯ В ЗАДАЧЕ УПРАВЛЕНИЯ ХАРАКТЕРИСТИКОЙ ЦИФРОВОГО ФИЛЬТРА ДЛЯ СПЕЦИАЛИЗИРОВАННОЙ КОМПЬЮТЕРНОЙ СИСТЕМЫ
}

Linearization in a digital filter control characteristic problem for a specialized computer system

Ухина А.В. ${ }^{1}$, к.физ-мат.н., доц. Яценко Т.П. ${ }^{2}$, д.т.н., проф. Ситников В.С. ${ }^{3}$ (Ukhina H.V., Yatsenko T.P., Sytnikov V.S.1)

Одесский национальный политехнический университет, г. Одесса

${ }^{1}$ E-mail: anyuta.uhina@inbox.ru

${ }^{2}$ E-mail: kuwtat@ukr.net

${ }^{3}$ E-mail: sitnvs@mail.ru

Copyright (C) 2014 by author and the journal "Automation technological and business - processes". This work is licensed under the Creative Commons Attribution International License (CC BY). http://creativecommons.org/licenses/by/4.0/

(c) (i) $\begin{aligned} & \text { ONAFT } \\ & \text { Open Access }\end{aligned}$

\section{Аннотация}

Определены зависимости, которые можно использовать для получения линейной характеристики управления АЧХ при аппроксимации характеристики. Показана возможность такого управления. 


\section{АЧХ при аппроксимации характеристики. Показана возможность такого управления.}

Ключевые слова

Аппроксимация, первичная обработка сигналов, метод «трубы», метод «веера», коэффициенты передаточной функции.

\section{Введение}

Специализированные компьютерные системы (СКС) активно внедряются в различные сферы жизнедеятельности человека. Они предназначены для сбора и обработки информации от датчиков, принятия решения и выработки управляющего воздействия на объект управления или соответствующие механизмы. Для повышения эффективности подобных систем необходимо управлять характеристиками перестраиваемых частотно-зависимых компонент. Например, в большинстве СКС имеются компоненты предварительной обработки и фильтрации входных сигналов, в состав которых наиболее часто входят цифровые фильтры. Для этой цели чаще всего рекомендуется использовать полиномиальные цифровые фильтры, отличительной особенностью которых является плоская амплитудно-частотная характеристика (АЧХ) в полосе пропускания. К их числу относятся известные фильтры Бесселя, Баттерворта и Чебышева второго рода [1-3].

За счет изменения коэффициентов числителя и знаменателя передаточной функции возможно как комплексное, так и раздельное управление характеристиками фильтра [4]. Однако в большинстве случаев для повышения их эффективности и плавного управления необходима линейная характеристика управления АЧХ устройства фильтрации. Обычно фильтры высокого порядка реализуются за счет соединения фильтров низкого порядка для обеспечения независимой перестройки характеристик.

\section{Изложение}

Анализ влияния коэффициентов передаточной функции цифрового фильтра на АЧХ проведен по передаточной функции первого порядка

$$
H(z)=\frac{a_{0} \pm a_{1} z^{-1}}{1+b z^{-1}},
$$

где $a_{0}, a_{l}, b$ - соответственно действительные коэффициенты числителя и знаменателя, \pm- в числителе соответствуют ФНЧ (+) и ФВЧ (-).

Для фильтров первого порядка коэффициенты числителя в общем случае равны $\left(\left|a_{0}\right|=\left|a_{1}\right|\right)$ и являются коэффициентом усиления $k=\left|a_{0}\right|=\left|a_{l}\right|$. Тогда передаточную функцию (1) для фильтра нижних частот (ФНЧ) можно записать в виде:

$$
H(z)=k \frac{1 \pm z^{-1}}{1+b z^{-1}}
$$

Из (2) следует, что линейное управление АЧХ возможно коэффициентом усиления $k$ за счет изменения коэффициентов числителя, что характерно для адаптивных фильтров. Однако, для уменьшения влияния шума на полезную составляющую сигнала при обработке необходима перестройка частоты среза фильтра.

В работах [4, 5] показаны возможные пути перестройки АЧХ ФНЧ и ФВЧ, а также трудности перестройки, вызванные тем, что коэффициенты числителя и знаменателя нелинейно зависят от частоты.

Для линеаризации характеристики управления АЧХ необходимо знание рабочего диапазона управления и заданной точности линеаризации. В большинстве случаев при высокой точности линеаризации диапазон управления разбивается на участки линейного управления, т.е. осуществляется кусочно-линейная аппроксимация. Полученная, таким образом, система линейных уравнений с ограничениями используется СКС для перестройки АЧХ цифрового фильтра.

Следует отметить, что при управлении цифровым фильтром необходимо осуществлять перестройку частоты среза с целью уменьшения влияния помех на полезную составляющую входного сигнала. В работе [5] получена зависимость нормированной частоты среза $\overline{\omega_{c}}$ ФНЧ в зависимости от коэффициента $b$ знаменателя передаточной функции фильтра Чебышева второго рода и уровня пульсаций в полосе задержания RS (в dB), рис. 1 


$$
\overline{\omega_{c}}=\arccos \left(-\frac{1-2 c^{2} \frac{1+b^{2}}{(1+b)^{2}}}{1-4 c^{2} \frac{b}{(1+b)^{2}}}\right),
$$

где $c^{2}=\frac{1}{\sqrt{10^{0.1 R S}}}, \overline{\omega_{c}}=2 \pi \frac{f}{f_{d}}, \overline{\omega_{c}} \in[0, \pi], f, f_{d}$ - соответственно текущая линейная частота и частота дискретизации.

В работе [5] для аппроксимации приведенных зависимостей разработан алгоритм, который основан на Чебышевской аппроксимации [6]. На основе анализа характера аппроксимируемой зависимости алгоритм выбирает направление аппроксимации либо с начала кривой, либо с ее конца.

Наиболее важным участком алгоритма аппроксимации считается блок, в котором происходит определение граничной точки между двумя участками аппроксимации. На каждой итерации происходит смещение конечной точки участка аппроксимации на одну позицию и заполнение матрицы размером $\mathrm{N} \times \mathrm{N}$ (где $\mathrm{N}$-количество точек, на которое разбивается участок, аппроксимируемой кривой) строками, каждая из которых содержит массивы точек для первого участка. Затем осуществляется пересчет коэффициента знаменателя $b$ и нормированной частоты среза $\overline{\omega_{c}}$, с целью получения расчетной погрешности и ее сравнения со значением заданной погрешности. Однако данный алгоритм обладает рядом недостатков: сложностью вычислений, большим объёмом занимаемой памяти, относительно длительным временем выполнения.

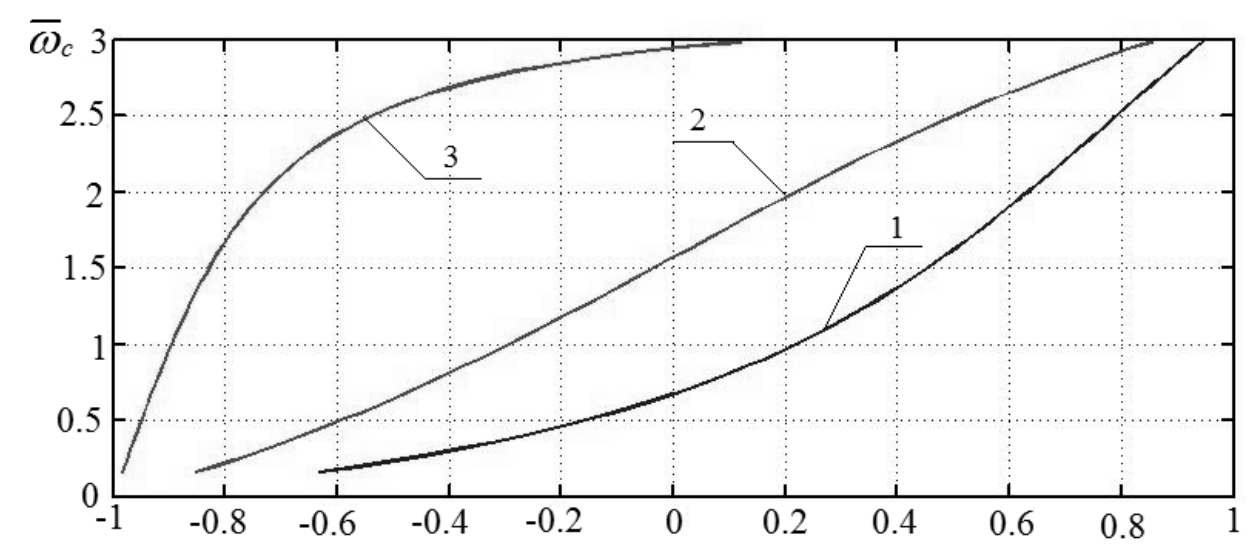

Рис. 1 - График зависимости частоты среза фильтра $\overline{\omega_{c}}$ от коэффициента $\boldsymbol{b}$ знаменателя передаточной функции фильтра Чебышева второго рода при уровнях пульсаций в полосе задержания $\mathrm{RS}=0,05 \mathrm{~dB}(1) ; 3 \mathrm{~dB}(2) ; 20 \mathrm{~dB}(3)$

Поэтому возникла необходимость рассмотреть алгоритмы аппроксимации, которые позволят упростить алгоритм аппроксимации и объем вычисления, а чтобы ускорить процесс получения линейных участков управления в зависимости от заданной погрешности.

В соответствии с Чебышевской аппроксимацией кривая на участке аппроксимации должна находиться внутри “трубы”, рис. 2. 


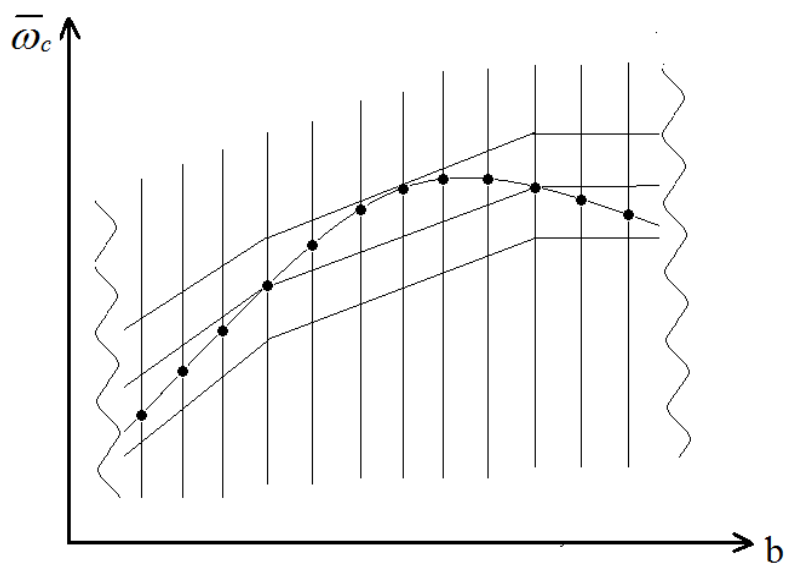

Рис. 2 - Графическое пояснение к Чебышевской аппроксимации по методу “трубы”

В этом случае можно указать границы “трубы” на участке аппроксимации и сравнивать значения исходной кривой с границами “трубы”, заменяя вычисления значений сравнением текущего значения с границами “трубы”. Тогда возникает задача вычисления границ “трубы”. Для этого нет необходимости анализировать характер аппроксимируемой зависимости, а начиная с первой точки строить аппроксимирующую прямую к последующим точкам. Последовательность действий в этом случае может быть такой.

На первом этапе между первой точкой участка аппроксимации и $n$-ой точкой строиться прямая линия. На величину погрешности “трубы” определяется верхняя и нижняя ее границы.

Следует отметить, что может быть два пути определение границ. Первый путь - вычисления верхней и нижней границы по тем же крайним точкам участка. Второй - прибавления к найденной прямой величину погрешности “трубы”. Отметим, что относительная погрешность “вычислительного” пути от “найденного” может доходить до $1 \%$.

На втором этапе осуществляется сравнение текущих значений с границами “трубы”. Если текущие значения находятся внутри “трубы” то берется следующее $n+1$-ое значение и процесс повторяется пока текущее значение кривой не превысит границы “трубы”. В этом случае процесс аппроксимации останавливается. Делается переход назад на одну точку (от $n+1$ к $n$ ) и осуществляется пересчет параметров аппроксимирующего участка.

Погрешность аппроксимации на участках показана на рис. 3 , при заданной относительной погрешности $\delta=0.01$. Алгоритм процесса аппроксимации по этому методу приведен на рис. 4.

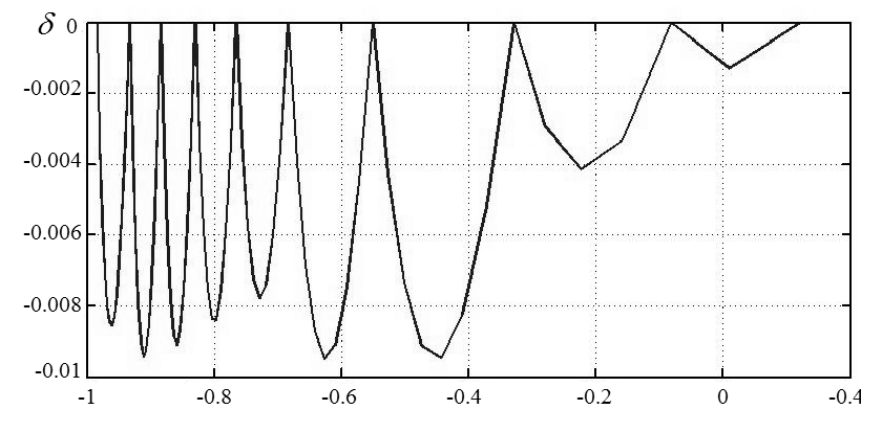

Рис. 3 - Зависимость относительной погрешности аппроксимации $\delta$ по методу “трубы” от коэффициента $\boldsymbol{b}$ знаменателя передаточной функции фильтра Чебышева второго рода при уровне пульсации в полосе задержания $\mathrm{RS}=20 \mathrm{~dB}$ 
АВТОМАТИЧНІ ТА АВТОМАТИЗОВАНІ СИСТЕМИ УПРАВЛІННЯ ТЕХНОЛОГІЧНИМИ ПРОЦЕСАМИ

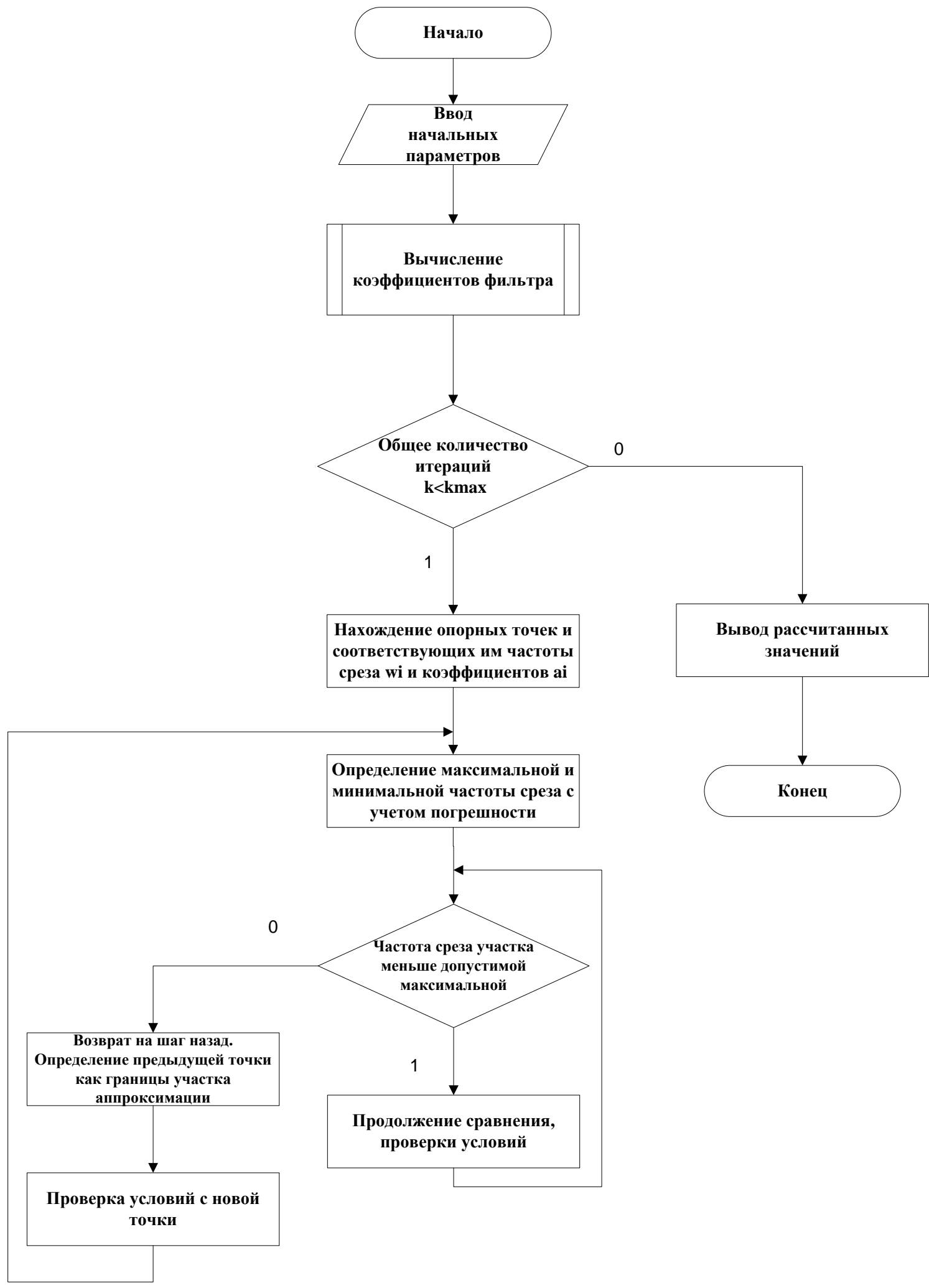

Рис. 4 - Алгоритм аппроксимации зависимости частоты $\overline{\omega_{c}}$ среза фильтра от коэффициента $\boldsymbol{b}$ знаменателя передаточной функции фильтра Чебышева второго рода по методу “трубы” 
В итоге аппроксимации получена система уравнений вида $y(k)=a_{0} x(k)+b_{0}$,

$$
\begin{cases}\bar{\omega}_{c}=9.8820 \cdot b+0.1571, & -0.9843<b \leq-0.9326, \\ \bar{\omega}_{c}=8.8745 \cdot b+0.6676, & -0.9326<b \leq-0.8840, \\ \bar{\omega}_{c}=7.2745 \cdot b+1.0996, & -0.8840<b \leq-0.8300, \\ \bar{\omega}_{c}=5.4879 \cdot b+1.4923, & -0.8300<b \leq-0.7656, \\ \bar{\omega}_{c}=3.8254 \cdot b+1.8457, & -0.7656<b \leq-0.6835, \\ \bar{\omega}_{c}=2.3645 \cdot b+2.1598, & -0.6835<b \leq-0.5506, \\ \bar{\omega}_{c}=1.2387 \cdot b+2.4740, & -0.5506<b \leq-0.3287, \\ \bar{\omega}_{c}=0.6357 \cdot b+2.7489, & -0.3287<b \leq-0.0816, \\ \bar{\omega}_{c}=0.3864 \cdot b+2.9060, & -0.0816<b \leq+0.1217,\end{cases}
$$

В данном методе приходиться дополнительно вычислять границы трубы и проверять попадания в нее текущих значений заданной кривой. Однако можно пойти другим путем, когда по ходу анализа вычисляются значения заданной относительной погрешности аппроксимирующих значений прямой и текущих значений кривой (метод “веера").

На первом этапе по этому методу также как и в предыдущем между первой точкой участка аппроксимации и $n$-ой точкой строиться прямая линия.

На втором этапе осуществляется вычисление относительной погрешности между значениями этой прямой и текущими значениями кривой. Если текущие значения имеют относительную погрешность меньше или равную заданной, то берется следующее $n+1$-ое значение и процесс повторяется пока для текущего значения кривой относительная погрешность будет больше заданной.

В этом случае процесс аппроксимации останавливается. Делается переход назад на одну точку (от $n+1 \kappa n$ ), осуществляется пересчет параметров аппроксимирующего участка. Погрешность аппроксимации на участках показана на рис. 5. Алгоритм процесса аппроксимации по методу “веера” показан на рис. 6.

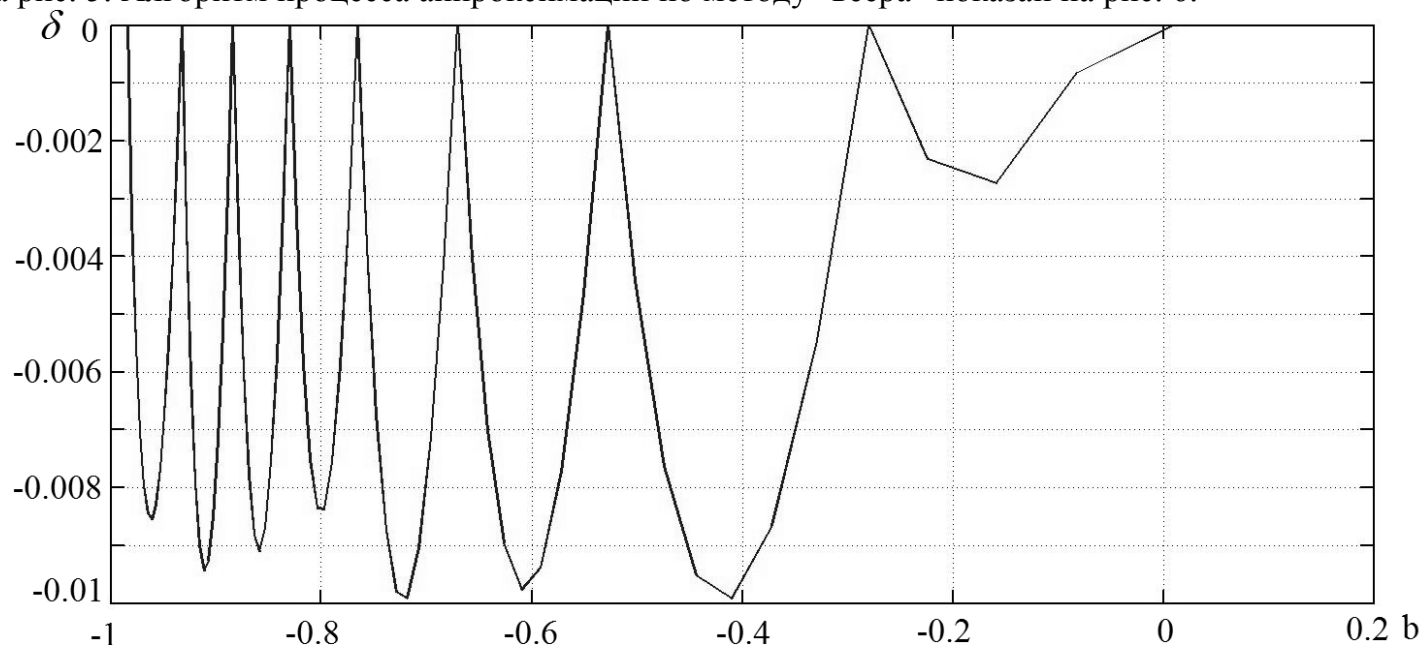

Рис. 5 - Зависимость относительной погрешности аппроксимации $\delta$ по методу “веера” от коэффициента $\boldsymbol{b}$ знаменателя передаточной функции фильтра Чебышева второго рода при уровне пульсации в полосе задержания $\mathrm{RS}=20 \mathrm{~dB}$ 
АВТОМАТИЧНІ ТА АВТОМАТИЗОВАНІ СИСТЕМИ УПРАВЛІННЯ ТЕХНОЛОГІЧНИМИ ПРОЦЕСАМИ

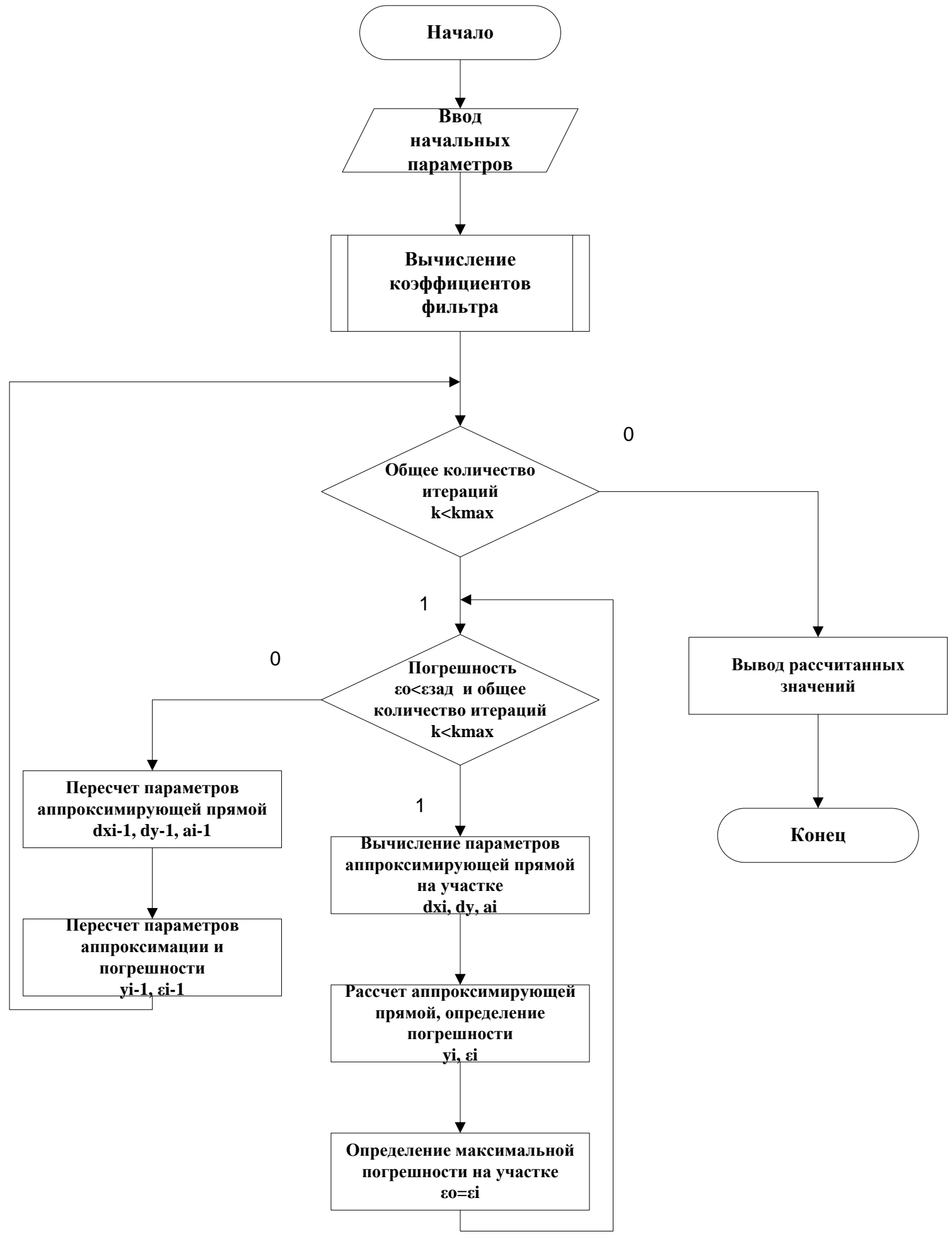

Рис. 6 - Алгоритм аппроксимации зависимости частоты среза $\overline{\omega_{c}}$ фильтра от коэффициента $\boldsymbol{b}$ знаменателя передаточной функции фильтра Чебышева второго рода по методу “веера” 
В итоге аппроксимации получена система уравнений аналогичная (1)

$$
\begin{cases}\bar{\omega}_{c}=9.8820 \cdot b+0.1571, & -0.9843<b \leq-0.9326, \\ \bar{\omega}_{c}=8.8745 \cdot b+0.6676, & -0.9326<b \leq-0.8840, \\ \bar{\omega}_{c}=7.2745 \cdot b+1.0996, & -0.8840<b \leq-0.8300, \\ \bar{\omega}_{c}=5.4879 \cdot b+1.4923, & -0.8300<b \leq-0.7656, \\ \bar{\omega}_{c}=3.7168 \cdot b+1.8457, & -0.7656<b \leq-0.6705, \\ \bar{\omega}_{c}=2.1972 \cdot b+2.1991, & -0.6705<b \leq-0.5275, \\ \bar{\omega}_{c}=1.1095 \cdot b+2.5133, & -0.5275<b \leq-0.2798, \\ \bar{\omega}_{c}=0.5826 \cdot b+2.7882, & -0.2798<b \leq+0.0101, \\ \bar{\omega}_{c}=0.3520 \cdot b+2.9452, & +0.0101<b \leq+0.1217,\end{cases}
$$

Сравнивая полученные результаты аппроксимации зависимости частоты среза фильтра $\overline{\omega_{c}}$ от коэффициента $b$ знаменателя передаточной функции фильтра Чебышева второго рода при уровнях пульсаций в полосе задержания $\mathrm{RS}=20 \mathrm{~dB}$ можно отметить, что с точностью до погрешности вычислений эти методы дают одинаковые результаты. Отличие начинается с пятой строки систем линейных уравнений (4) и (5), где сказываются вычислительные погрешности.

За счет оптимизации второго алгоритма можно упростить вычисления и ускорить процесс нахождения системы линейных уравнений при заданной погрешности аппроксимации $\delta$.

На рис. 7 представлены АЧХ, построенные по формуле и аппроксимированным значениям коэффициентов $k$ и $b$ фильтра. Для уменьшения погрешности необходимо увеличить количество участков аппроксимации для достижения заданной точности воспроизведения АЧХ при ее перестройке.

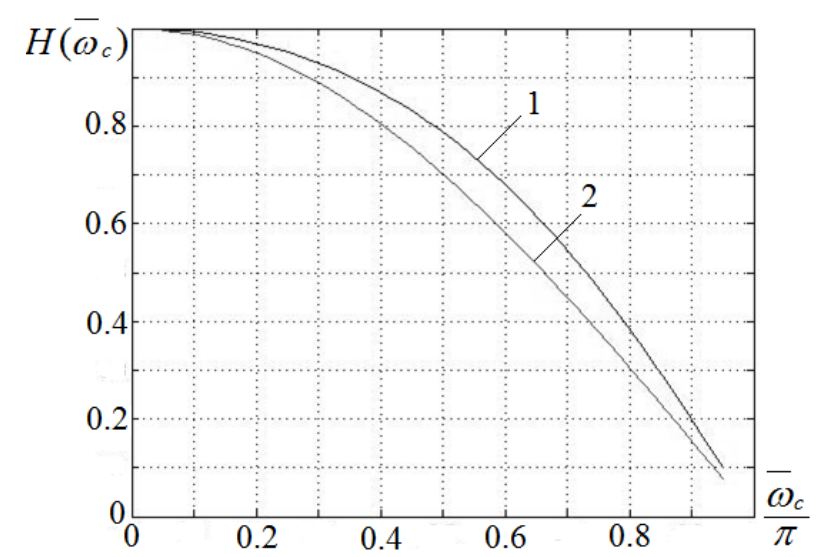

Рис. 7 - Графики АЧХ построенные по формуле (1) и аппроксимированным (2) значениям коэффициентов $\boldsymbol{k}$ и $\boldsymbol{b}$

Заключение фильтра

Таким образом, в результате аппроксимации получены системы линейных уравнений, описывающие характеристику управления, кроме того показана возможность такого управления. 


\title{
АВТОМАТИЧНІ ТА АВТОМАТИЗОВАНІ СИСТЕМИ УПРАВЛІННЯ ТЕХНОЛОГІЧНИМИ
} ПРОЦЕСАМИ

Литература

[1] Illiev G. Efficient design of adaptive complex narrowband IIR filters / Illiev G., Nikolova Z., Stoyanov G., Egiazarian K. / XII European Signal Processing Conference "EUSIPCO-2004" / 6-10 Sept., 2004, Vienna, Austria - p.p. 15971600.;

[2] Устройство адаптивной фильтрации речевых сигналов “Золушка-микро-3“ http://speetech.by/manuals/manual_zolushka_micro3.pdf;

[3] Сергиенко А.Б. Цифровая обработка сигналов / А.Б. Сергиенко - СПб.: Питер, 2006. — 751 с.;

[4] Букашкин С.А. Справочник по расчету и проектированию ARC-схем; Под ред. А.А. Ланнэ — / C.A. Букашкин, В.П.Власов, Б.Ф.Змий и др. М. Радио и связь, 1984. - 368 с.;

[5] Дикусар Е.В. Аппроксимация характеристики управления полиномиальной компонентой первого порядка / Е.В. Дикусар, А.А. Швец, Г.А.Грицкевич// Праці одеськ.політехн. ун-та - 2011 - Вип.1(35) - С. 141-146.;

[6] Литовченко Н.М. Анализ критериев аппроксимации амплитудно-частотной характеристики устройства / Н.M. Литовченко, В.С. Ситников, А.В. Яковлев // Холодильна техніка і технологія. — 2006. — № 1(99). — С. $86-$ 88.;

References

[1] Illiev G. Efficient design of adaptive complex narrowband IIR filters / Illiev G., Nikolova Z., Stoyanov G., Egiazarian K. / XII European Signal Processing Conference "EUSIPCO-2004" / 6-10 Sept., 2004, Vienna, Austria - p.p. 15971600 ;

[2] Ustroistvo adaptivnoi fil'tratsii rechevykh signalov "Zolushka-mikro-3" http://speetech.by/manuals/manual_zolushka_micro3.pdf;

[3] Sergienko A.B. Tsyfrovaia obrabotka signalov / A.B. Sergienko - SPB.: Piter, 2006. - 751 s.;

[4] Bukashkin S.A. Spravochnik po raschetu i proektirovaniiu ARC-skhem; Pod. red. A.A. Lanne - / S.A. Bukashkin, V.P. Vlasov, B.F. Zmii i dr. M. Radio I sviaz', 1984. - 368 s.;

[5] Dikusar E.V. Approksimatsiia kharakteristiki upravleniia polynomial'noi komponentoi pervogo poriadka / E.V. Dikusar, A.A. Shvets, G.A. Gritskevich // Pratsi odes'k.politekhn.un-ta - 2011 - Vyp.1(35) - S. 144-146;

[6] Litovchenko N.M. Analiz kriteriev approksimatsii amplitudno-chastotnoi kharakteristiki ustroistva / N.M. litovchenko, V.S. Sitnikov, A.V. Iakovlev // Kholodyl’na tekhnika i tekhnologiia. - 2006. - №1(99)ю - S. 86-88.

\section{АВТОМАТИЗАЦІЯ АНАЛІЗУ КАР'СРНОГО РОЗВИТКУ ТА ПІДТРИМКА ПРИЙНЯТТЯ РІШЕНЬ ЩОДО АТЕСТАЦЇ̈ РОЗРОБНИКІВ ПРОГРАМНОГО ЗАБЕЗПЕЧЕННЯ}

\author{
Водолазкіна К.О., аспірантка ${ }^{1}$ \\ 1апорізький національний технічний університет, м. Запоріжжя \\ Copyright (C) 2014 by author and the journal "Automation technological and business - processes". \\ This work is licensed under the Creative Commons Attribution International License (CC BY). \\ http://creativecommons.org/licenses/by/4.0/
}

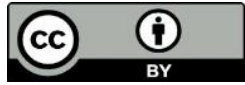

ONAFT

Open Access 